\title{
ON THE LOGARITHMIC COEFFICIENTS OF CLOSE-TO-CONVEX FUNCTIONS
}

\author{
M. M. ELHOSH \\ (Received 20 May 1991; revised 7 April 1992) \\ Communicated by P. C. Fenton
}

\begin{abstract}
Logarithmic coefficient bounds for some univalent functions are given in this paper.

1991 Mathematics subject classification (Amer. Math. Soc.): 30C45, 30C50, $30 \mathrm{C55}$.
\end{abstract}

Let $S$ denote the usual class of univalent functions $f(z)$ normalized so that $f(0)=$ $f^{\prime}(0)-1$ in $|z|<1$. Let $C$ denote the set of functions $f(z)$ normalized as above and which satisfy the condition $\operatorname{Re} z f^{\prime}(z) / g(z) \geq 0$ in $|z|<1$, where $g(z)$ itself is subject to the conditions $\operatorname{Re} z g^{\prime}(z) / g(z) \geq 0, g(0)=0$ and $\operatorname{Re} g^{\prime}(0)>0$ in $|z|<1$. Then $f(z)$ is called close-to-convex relative to the starlike function $g(z)$. We denote this set of functions $g(z)$ by $S^{*}$. Now let $K$ denote the set of functions $Q(z)$ which satisfy the conditions: $Q(0)=0, \operatorname{Re} Q^{\prime}(0)>0$ and $\operatorname{Re}\left(z Q^{\prime \prime} / Q^{\prime}+1\right) \geq 0$ in $|z|<1$. Such $Q(z)$ are called convex. It is well known that each $z Q^{\prime}(z) \in S^{*}$ and $K \subset S^{*} \subset C \subset S$ (see for example [6, pp. 40-46]; [11, pp. 11-18]; [14, p. 361]).

Now, as in [6, p. 151], each $f(z) \in S$ has a logarithmic expansion

$$
\log \frac{f(z)}{z}=2 \sum_{n=1}^{\infty} \gamma_{n} z^{n}
$$

in $|z|<1$ where $\gamma_{n}$ are known as the logarithmic coefficients. The problem of the best upper bounds for $\left|\gamma_{n}\right|$ is still open. In fact even the proper order of magnitude is still not known. It is known, however, for the starlike functions that the best bound

(C) 1996 Australian Mathematical Society 0263-6115/95\$A2.00+ 0.00 
is $\left|\gamma_{n}\right| \leq 1 / n \quad(n \geq 1)$ and that this is not true in general [6, p. 151]; [5, p. 898]; [1, p. 140] and [7].

The importance of this problem in relation to the Bieberbach conjecture was pointed out by Milin in his conjecture (see [1, p. 141]; [6, pp. 155-156]; [5, p. 899]) that

$$
\sum_{m=1}^{n} \sum_{k=1}^{m}\left(k\left|\gamma_{k}\right|^{2}-\frac{1}{k}\right) \leq 0 \text {, }
$$

which led De Branges, by proving this conjecture, to the proof of the Bieberbach conjecture [2]. Milin has shown [6, p. 151] that $\sum_{k=1}^{n}\left(k\left|\gamma_{k}\right|^{2}-1 / k\right) \leq \delta$ where $\delta<0.312$.

It is known that $\delta$ cannot be reduced to zero in general $[6$, p. 155]. However, its exact value remains unknown. In this note we show that $\left|\gamma_{n}\right| \leq 1 / n \quad(n \geq 1)$, for $f \in C$, and consequently $\delta=0$ as for the starlike functions. We shall use $\sum a_{n} z^{n} \ll \sum b_{n} z^{n}$ to mean $\left|a_{n}\right| \leq b_{n}$ for $n \geq 1[12$, p. 52] and $f \prec F$ to mean that $f(0)=F(0)$ and $f(z:|z|<1) \subset F(z:|z|<1)$ or equivalently $f(z)=F(\phi(z))$, where $\phi(0)=0$ and $|\phi(z)|<1[6$, p. 190].

THEOREM 1. Let $f \in C$ so that (1) holds. Then for $n \geq 1$ we have

$$
\left|\gamma_{n}\right| \leq \frac{1}{n}
$$

ProOF. For $f \in C$ let $\left\{f_{k}\right\}$ be a sequence in $C$ which converges uniformly on compact subsets to $f \in C$. Let also for a fixed $n, J(g)=\left|\gamma_{n}\right|$ where $g(z)=$ $\log (f(z) / z)=2 \sum_{n=1}^{\infty} \gamma_{n} z^{n}$ and let $\log \left(f_{k}(z) / z\right)=2 \sum_{n=1}^{\infty} \gamma_{n}^{(k)} z^{n}$. Then, using the coefficient formula we deduce for $z=r e^{i \theta}, 0<r<1$, that

$$
\begin{aligned}
|| \gamma_{n}^{(k)}|-| \gamma_{n}|| & \leq\left|\gamma_{n}^{(k)}-\gamma_{n}\right| \\
& =\left|\frac{1}{2 \pi i} \int_{|z|=r}\left(\log \frac{f_{k}(z)}{z}-\log \frac{f(z)}{z}\right) \frac{d z}{z^{n+1}}\right| \\
& \leq r^{-n} \max _{|z|=r}\left|\log \left(f_{k}(z) / f(z)\right)\right| \\
& \rightarrow 0,
\end{aligned}
$$

since $f_{k} \rightarrow f$ uniformly on $|z|=r$ as $k \rightarrow \infty[11, \mathrm{p}$. 40]. Thus we see that $\left|\gamma_{n}^{(k)}\right|^{2} \rightarrow\left|\gamma_{n}\right|^{2}$ so that $J(g)$ is continuous.

Now let $h(z)=\log \left(f_{1}(z) / z\right)=2 \sum_{n=1}^{\infty} \gamma_{n}^{\prime} z^{n}, \phi(z)=\log \left(f_{2}(z) / z\right)=2 \sum_{n=1}^{\infty} \gamma_{n}^{\prime \prime} z^{n}$ in $|z|<1$ where $f_{1}, f_{2} \in C$. Let also $g=t h+(1-t) \phi, 0 \leq t \leq 1$. Then by [11, Lemma 5.6] we have

$$
\begin{aligned}
J(g)=\left|\gamma_{n}\right|^{2} & =\left|t \gamma_{n}^{\prime}+(1-t) \gamma_{n}^{\prime \prime}\right|^{2} \\
& \leq t\left|\gamma_{n}^{\prime}\right|^{2}+(1-t)\left|\gamma_{n}^{\prime \prime}\right|^{2} \\
& =t J(h)+(1-t) J(\phi),
\end{aligned}
$$


which implies that $J(g)$ is convex.

Thus, in view of [11, Theorem 4.6], we need only prove Theorem 1 for the extreme points of the closed convex hull of $C$, denoted by $E H C$, since $\max \{J(f): f \in E H C\}$ $=\max \{J(f): f \in C\}$ in this case.

Functions $f \in E H C$ are of the form

$$
f(z)=\left(z-\frac{1}{2}(x+y) z^{2}\right) /(1-y z)^{2}
$$

where $x \neq y$ and $|x|=|y|=1$.

With a suitable rotation (see $[11$, p. 83]), this can be written in the form

$$
f(z)=\left(z-b z^{2}\right) /(1-z)^{2}
$$

where $|b-1 / 2|=1 / 2$.

Writing $\psi(z)=(1-b z) /(1-z)$ we see that

$$
\operatorname{Re} \psi(z)=\frac{1}{2}+\frac{1}{2}\left(\frac{1+(1-2 b) z}{1-z}\right) \geq \frac{1}{2} .
$$

We also see that $\operatorname{Re}\left(z \psi^{\prime \prime} / \psi^{\prime}+1\right)=\operatorname{Re}((1+z) /(1-z)) \geq 0$ which implies that $\psi$ is convex and consequently starlike of order $1 / 2$ (see $[4$, p. 418];[6, p. 251]). Thus, as in [4, p. 417] and [16, p. 722], using Herglotz's formula (see [6, pp. 22-40]; [11, pp. 27-30])

$$
p(z)=\int_{0}^{2 \pi}\left(\frac{1+e^{-i t} z}{1-e^{-i t} z}\right) d \mu(t)
$$

where $p(0)=1, \operatorname{Re} p(z) \geq 0, d \mu(t) \geq 0$ and $\int_{0}^{2 \pi} d \mu(t)=1$, we obtain

$$
\begin{aligned}
\log \psi(z) & =\int_{0}^{2 \pi} \log \left(\frac{1}{1-z e^{-i t}}\right) d \mu(t) \\
& =\sum_{n=1}^{\infty}\left(\int_{0}^{2 \pi} e^{-i n t} d \mu(t)\right) \frac{z^{n}}{n} \\
& \ll \sum_{n=1}^{\infty} \frac{z^{n}}{n}=\log \frac{1}{1-z} .
\end{aligned}
$$

Hence we see that

$$
\begin{aligned}
\log \frac{f(z)}{z} & =\log \frac{1}{1-z}+\log \frac{1-b z}{1-z} \\
& \ll 2 \log \frac{1}{1-z} .
\end{aligned}
$$

This gives (2) by the definition of $\ll$ above. 
COROLLARY 1. For $f \in C$ we have

$$
\begin{aligned}
\frac{1}{2 \pi} \int_{0}^{2 \pi}\left|\frac{z f^{\prime}(z)}{f(z)}\right|^{2} d \theta & =1+4 \sum_{n=1}^{\infty} n^{2}\left|\gamma_{n}\right|^{2} r^{2 n} \\
& \leq 1+4 \sum_{n=1}^{\infty} r^{2 n} \\
& =\frac{1}{2 \pi} \int_{0}^{2 \pi}\left|\frac{z K^{\prime}(z)}{K(z)}\right|^{2} d \theta
\end{aligned}
$$

where $z=r e^{i \theta}, 0<r<1$, and $K(z)=z(1-z)^{-2}$.

THEOREM 2. For $f \in E H C$ we have

$$
\log \frac{f(z)}{z} \prec \log \frac{K(z)}{z},
$$

or equivalently

$$
\frac{f(z)}{z} \prec \frac{K(z)}{z}
$$

where $K(z)$ is as defined above.

PROOF. This theorem follows from the fact that $\operatorname{Re}((1-b z) /(1-z)) \geq 1 / 2$ is equivalent to $(1-b z) /(1-z) \prec 1 /(1-z),[11$, p. 53], and this is equivalent to $\log ((1-b z) /(1-z)) \prec \log (1 /(1-z))[17$, p. 23]. Thus we have

$$
\begin{aligned}
\log \frac{f(z)}{z} & =2\left(\frac{1}{2} \log \frac{1}{1-z}+\left(1-\frac{1}{2}\right) \log \frac{1-b z}{1-z}\right) \\
& \prec 2 \log \frac{1}{1-z}
\end{aligned}
$$

as required.

COROllaRY 2. For $f \in E H C$ we see from [11, Theorem 3.3]; [6, Theorem 6.1] and (4) that

$$
\int_{0}^{2 \pi}\left|\log \frac{f(z)}{z}\right|^{q} d \theta \leq \int_{0}^{2 \pi}\left|\log \frac{K(z)}{z}\right|^{q} d \theta
$$

where $z=r e^{i \theta}, 0<r<1$, and $q>0$. This extends [10, Theorem 1] for $f \in E H C$.

CoRollary 3. For $f \in C$ and $\gamma_{n}$ as defined in Theorem 1 we see from $[6$, p. 212 (Exercise 7)], (4) and (2) that

$$
\sum_{k=1}^{n} k\left|\gamma_{k}\right|^{2} \leq \sum_{k=1}^{n} 1 / k
$$


THEOREM 3. Let $f \in C$ and $f(z)=z+a_{2} z^{2}+a_{3} z^{3}+\cdots$. Then for $n \geq 2$ we have

$$
|| a_{n}|-| a_{n-1}|| \leq 1
$$

PROOF. For some choice of $\xi$ on the boundary, with $|\xi|=1$, we have

$$
(1-\xi z) \frac{f(z)}{z}=\exp \left\{\sum_{n=1}^{\infty}\left(2 \gamma_{n}-\frac{\xi^{n}}{n}\right) z^{n}\right\}
$$

Applying the Lebedev-Milin inequality $\left|\beta_{n}\right|^{2} \leq \exp \left\{\sum_{k=1}^{n} k\left|\alpha_{k}\right|^{2}-\sum_{k=1}^{n} 1 / k\right\}$ for the expansion $\sum_{k=0}^{\infty} \beta_{k} z^{k}=\exp \left\{\sum_{k=1}^{n} \alpha_{k} z^{k}\right\}, \quad \beta_{0}=1[6$, p. 143]; [5, p. 897], we deduce by using the triangle inequality that

$$
|| a_{n}|-| a_{n-1}|| \leq \exp \left\{2 \sum_{k=1}^{n}\left(k\left|\gamma_{k}\right|^{2}-\operatorname{Re}\left(\xi^{k} \gamma_{k}\right)\right)\right\} .
$$

We now write $\xi=e^{i t}$ and choose $t$ such that $k t+\arg \left(\gamma_{k}\right)=0$. We see that $e^{k i t} \gamma_{k}=\left|\gamma_{k}\right|$. Using this and (2) in (5) we deduce Theorem 3 since

$$
\sum_{k=1}^{n}\left(k\left|\gamma_{k}\right|^{2}-\operatorname{Re}\left(\xi^{k} \gamma_{k}\right)\right)=\sum_{k=1}^{n}\left(k\left|\gamma_{k}\right|^{2}-\left|\gamma_{k}\right|\right) \leq 0 .
$$

REMARK 1 . The case $n=3$ has been proved by Koepf [13]. For the full class $S$ the author $\left[9\right.$, p. 13] obtained ||$a_{3}|-| a_{2}||<1.411$.

REMARK 2. It has been shown by Pearce [15] that functions of the form (3) are extreme points of $S$ whenever $0<|\arg (-x / y)| \leq \pi / 4$ and consequently Theorems 2 and 3 hold for these functions. Our results give a partial answer to the questions raised in $[8]$ and $[3$, Problem $6.71 ;$ p. 558].

REMARK 3. The Koeke function $K(z)=z(1-z)^{-2}$ and its rotations show that our results are the best possible.

REMARK 4. Let $\left(z f^{\prime} / f\right)^{q}=1+\sum c_{n}(q) z^{n}$ and $[(1+z) /(1-z)]^{q}=1+\sum D_{n}(q) z^{n}$ where $n, q$ are positive integers. Then we have $\left|c_{n}(q)\right| \leq D_{n}(q)$ since $\left(z f^{\prime} / f\right)^{q} \ll$ $[(1+z) /(1-z)]^{q}$ in this case. (See $[12$, Lemma 2.4 .1$, p. 53].) We now easily see that

$$
\int_{0}^{2 \pi}\left|\frac{z f^{\prime}(z)}{f(z)}\right|^{2 q} d \theta \leq \int_{0}^{2 \pi}\left|\frac{z K^{\prime}(z)}{K(z)}\right|^{2 q} d \theta
$$

and this extends Corollary 1 when $q$ is a positive integer. 
The author would like to thank the referees for their useful comments to improve the first version of this paper.

\section{References}

[1] J. Anderson, K. Barth and D. Brannan, 'Research problems in complex analysis', Bull. London Math. Soc. 9 (1977), 129-162.

[2] L. De Branges, 'A proof of the Bieberbach Conjecture', Acta Math. 154 (1985), 137-152.

[3] D. Brannan and J. Clunie, Aspects of contemporary complex analysis (Academic Press, 1980).

[4] L. Brickman, D. Hallenbeck and T. MacGregor, 'Convex hulls and extreme points of families of starlike and convex mappings', Trans. Amer. Math. Soc. 185 (1973), 413-428.

[5] P. Duren, 'Coefficients of univalent functions', Bull. Amer. Math. Soc. (5) 83 (1977), 891-911.

[6] _- Univalent functions (Springer, New York, 1983).

[7] P. Duren and Y. Leung, 'Logarithmic coefficients of univalent functions', J. Analyse Math. 36 (1979), 36-43.

[8] M. M. Elhosh, 'On the logarithmic coefficients of close-to-convex functions', Math. Japon. (2) 31 (1986), 201-204.

[9] — ' 'On mean p-valent functions', Rev. Roumaine Math. Pures Appl. 34 (1989), 11-15.

[10] D. Girela, 'Integral means and BMOA norms of logarithms of univalent functions', $J$. London Math. Soc. 33 (1986), 117-132.

[11] D. Hallenbeck and T. MacGregor, Linear problems and convexity techniques in geometric function theory (Pitman, London, 1984).

[12] E. Hille, Ordinary differential equations in the complex domain (Wiley, New York, 1976).

[13] W. Koepf, 'On the Fekete-Szego problem for close-to-convex functions', Proc. Amer. Math. Soc. 101 (1987), 89-95.

[14] T. MacGregor, 'Applications of extreme point theory to univalent functions', Michigan Math.J. 19 (1972), 36I-376.

[15] K. Pearce, 'New support points of S and extreme points of KS', Proc. Amer. Math. Soc. 81 (1981), 425-428.

[16] B. Pinchuk, 'On starlike and convex functions of order $\alpha$ ', Duke Math. J. 35 (1968), 721-734.

[17] R. Robinson, 'On the theory of univalent functions', Ann. of Math. 37 (1936), 1-35.

Department of Mathematics

The University College of Wales

Aberystwyth

Dyfed

UK 